\title{
A conversation with Christine Seidman
}

\begin{abstract}
D r. Christine Seidman of the Harvard Medical School has uncovered the genetic basis of many human cardiovascular disorders, from cardiomyopathy and heart failure to congenital heart malformations. Here, Seidman (Figure 1) speaks about her early intrigue with atrial natriuretic factor and her more current gene-intensive investigations. The full interview, with many more stories about her interest in the ear, an early inspirational patient, and her thoughts on work-life balance, can be seen on the JCI website, http://www.jci.org/ kiosk/cgm.
\end{abstract}

JCI: Can you start by telling us where you grew up, about your parents, and about your early interests in science?

Seidman: I was born in Kalamazoo, Michigan, but I moved because my father was transferred to New York, and I grew up on Long Island. Subsequently, I went to high school there and went on to college at Harvard University, and then went on medical school at George Washington in DC. I moved back north to Baltimore to do my internship and residency at Johns Hopkins and Cardiology Fellowship at the Mass General Hospital. I was very interested in science as a child, but I didn't have any exposure to people doing science at a professional level. In fact, the closest I came to seeing individuals who were really devoted to science as a career were physicians. We had a general practitioner who would come to our home and visit when one of us was ill. I think that certainly was the first connection that I made that suggested that science might not just be about bugs and butterflies and things I could dissect under a microscope, but could be actually about living people.

JCI: Did you decide early on that you wanted to focus on science or being a physician?

Seidman: I think it was probably a little bit of both. Having no direct exposure to people doing scientific investigation, early on I assumed that science and medicine were one and the same. And I actually think that's still true. I think it's the foundation of good medicine.

JCI: When you were doing your residency at Hopkins, you chose Victor McKusick as one of your early mentors. Did he turn you onto genetics or were you already on that path?
Seidman: I was not even aware of what genetics was, or how it could be applicable to human disease or basic scientific discovery, in those years. McKusick was an incredible individual, a brilliant scientist, and an amazing mentor. He took the chance of taking people who had come through traditional medical approaches and saying, "Maybe you should think about science." And the approach that he took was very personal. He invited us to his home. We would be well fed, something quite remarkable during internship and residen$c y$, and then we would discuss a scientific manuscript. Those discussions were lively and interactive. They were not stilted or anything threatening, and one could ask questions and really begin to explore. $\mathrm{He}$ was a geneticist and that certainly made me think about using genetics in some way.

JCI: Atrial natriuretic factor: the structure, the function, and the sequence. It overwhelms the beginning part of your CV. What was that time like when you were on the hunt and when you were first working on ANF?

Seidman: The way I came to work on ANF was rather unusual. I had been a cardiac fellow and doing research in the laboratories of Charles Homcy and Robert Graham. Those two wonderful scientists and mentors were devoted to trying to understand adrenergic receptors.

I had been toiling away without a whole lot of success. When I came home on a Sunday afternoon and read an article in Science that described the protein analysis of a small molecule, about 21 amino acids that encoded what appeared to be a diuretic that was made by the atria of this heart. Any cardiologist would be interested in that and so my interest was really piqued. The senior author on that paper was Phil Needleman - then at Washington University, St. Louis - and he had used hundreds of rodents to purify the peptide to get sufficient quantities to be able to deduce the amino acid sequence. Using molecular approaches, I knew that one could take that protein sequence information, produce degenerate oligonucleotide probes and screen a library to clone a particular cDNA, and subsequently the gene. I had been floundering away trying to do this kind of science in the adrenergic system, but didn't have that critical piece of infor- mation, the amino acid sequence. Needleman's partial peptide sequence of ANF provided the seminal piece of information that I was lacking in the adrenergic system. So I made a library from several rodent atria, from which I isolated and sequenced the ANF cDNA, and then the ANF gene.

JCI: Do you still work on ANF at all?

Seidman: Not so very much. ANF is one of the molecules that is reactivated and expressed in response to all sorts of cardiac pathologies including the cardiomyopathies. It's one of the most profoundly upregulated molecules during cardiac remodeling that occurs in hypertrophy and heart failure. But we now monitor it more as a marker rather than having an interest in its actual physiologic and pathologic processes.

JCI: Your more recent work has looked into mutations in various genes that cause anything from heart failure to cardiomyopathy. How many has your lab found over the years?

Seidman: We don't keep a running scorecard. We know that all of the cardiomyopathies, and indeed the majority of congenital heart defects that have a genetic etiology, are profoundly heterogeneous. Perhaps more important than the actual number of genes is whether that knowledge points to a shared function that is disturbed by mutation. For example, mutations that cause hypertrophic cardiomyopathy all participate in contractile functions of the heart. Mutations that cause congenital heart disease participate in regulating gene expression during heart development. Those insights are more luminary of mechanism than just numbers of genes and numbers of mutations. But yes, we have discovered scores of genes and hundreds of individual mutations.

JCI: Have any of them come as a surprise? Seidman: Absolutely. Early on, we made assumptions about the mechanisms by which a heart cell could increase in size because they are largely terminally differentiated so they don't replicate. One doesn't get hyperplasia of the heart. Instead, one gets an increase in the size of the heart, and when thinking about that from 30,000 feet, you might expect that genes that are involved in growth would be activated in the setting of those diseases. Since cancer biologists have discovered lots of genes 
that promote cell growth, we assumed that there could be activation of oncogenes that would cause heart cells to increase in size. I was shocked when we learned that a simple set of proteins involved in contraction could actually trigger the heart to grow in size. We're making some progress in understanding why, but I don't think any of us thought that mutations in proteins that are involved in force production would trigger growth of the myocyte and increase the proliferation of cardiac fibroblasts throughout the entire heart.

JCI: In order to find some of these mutations, you have relied on finding large pedigrees. How much of this starts with the families? How open are they to being studied?

Seidman: Families with diseases, in particular those with lots of symptoms and those that impact survival, are amazingly receptive and are great advocates of getting physician-scientists to study their condition, often with the conviction that this will promote discovery of cures. We don't often get to the cure stage, but we can't get there without knowing the root cause and these families have been amazingly helpful.

When we explain that we'd like to evaluate an affected individual and their relatives, both those with and without the condition, many families offer to orchestrate an event to bring their family together at one time. This turns out to be profoundly informative because in that scenario one can see the full spectrum of clinical manifestations - from young people who we will subsequently learn might carry a gene defect and have no manifestation, all the way through to the individuals who are profoundly affected by a disease. It's an amazing opportunity in a very short amount of time to see a spectrum of disease that could take decades to learn about if one saw only individual patients.

JCI: How do the families react when you tell them that you have found the mutation that might lead to an explanation for what it is that's happening to them?

Seidman: Individuals who are affected are usually not surprised because they know they have a clinical disease and their initial response is often, "How do we fix it?" It sometimes takes a little more time for the affected individual to recognize that having a genetic cause also implies they can transmit the mutation to their offspring. That can be a very hard and difficult discussion. We've seen some families who accept that, until a precise cause for their disease was

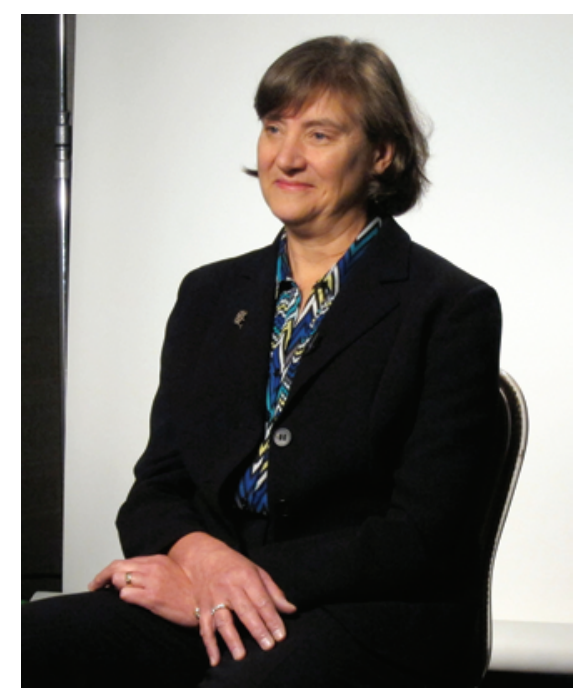

Figure 1

Christine Seidman on April 27, 2013, in Chicago, Illinois. Image credit: Karen Guth.

discovered, they had no opportunity to prevent that transmission. Other families are just devastated by this recognition of heritability. We try to support these families in many different ways, but there is no question that the responses are highly variable. Over time, the vast majority of people who learn that they either do or do not carry a pathogenic mutation that causes a disease in themselves, or in family members, are glad that they've chosen to know and learn this information. But this is a deeply personal decision. One of the good things about studying families is that often you will find an elder family member - a grandparent, a great aunt, or someone - who has lived a long and fulfilled life even with the disease. It is those individuals that I point to when speaking with a family who now knows their child will develop the disease with high likelihood, because even for really serious diseases, individual outcomes are different. There is a lot we don't understand about what influences disease expression, even when there is a genetic cause.

JCI: Given these complexities, why not just work in mice?

Seidman: First and foremost, I'm a physician. I'm not a mouse doctor, although I do have several of those small four-legged patients as well. My motivation for science still remains deeply entrenched in my interests in improving the lives of people around me, and improving their ability to have healthy, productive, and as long and happy a life as is possible. The mice are another mechanism to explore things that we can't do in people. The mice provide opportunities for us to remove the heart, to study different signals that are activated by the heart that we simply cannot get from our human patients.

JCI: You landed at Harvard fairly early, but you've stayed for your whole career. What has that environment provided to you that has kept it so tantalizing?

Seidman: I went to Harvard as an undergraduate and I came back as a faculty member years later primarily because of personal issues. I am part of a duo. During my internship and residency training, my husband and scientific partner was at the $\mathrm{NIH}$. And so we had this 50-mile commute each day in each direction that made life more complicated than need be. When he was recruited to Harvard, it was an opportunity for us to consolidate our lives into the same city.

One of the most important reasons for staying is having talented individuals who come from all over the world to work with us at Harvard. The medical students, the postdoctoral fellows, and senior scientists are smart, they're creative, and they really drive science in ways that makes it exciting for everybody around them. I'm sure those same individuals are at many other institutions, but the ones that we've had the privilege to work with have just really made us say, "Why leave?"

JCI: If you were to leave, what would you do? Or what would you have done if you weren't a doctor?

Seidman: Most likely I would continue to work in human genomics. I still want to understand what causes human diseases, to understand the mechanisms for different clinical expression, and to find better ways to treat them. I'd like to see human genomics broadly applied in medicine because I believe that this will improve the way we think about disease and treatments. If I completely switched fields, I might go into botany. A long time ago, I wanted to understand how we could grow crops that would feed people in places where fertilizer, and all sorts of access to the kinds of things that happen in agriculture in the United States, were unavailable. And I worked on projects to provide nitrogen fixation in Rhizobium plants to allow them to have intrinsic fertilizer. I love to garden and perhaps I would go and do something related to botany on a more global issue.

\section{Ushma S. Neill}

